\title{
USO DEL ARCO EN LAS COHORTES PRETORIANAS
}

\author{
USE OF THE BOW BY THE PRAETORIAN COHORTS
}

POR

\author{
HÉCtor Ceñal Martínez*
}

\section{RESUMEN - ABSTRACT}

A lo largo de este artículo hemos realizado una aproximación al uso de un arma que normalmente no se asocia con los soldados de la guardia pretoriana, mostrando así cómo los nuevos tiempos obligaron a estos soldados a adaptarse. Para ello hemos recurrido tanto a las fuentes clásicas como a la epigrafía. Así podemos aseverar sin duda el empleo de esta arma en el pretorio durante el siglo III d. C., como consecuencia de los nuevos modos de combate ante enemigos nuevos y viejos..

In this article we have attempted to present a general idea of the use of a weapon that is not usually associated with Praetorian soldiers, showing how soldiers were forced to adapt to new times. We have analyzed epigraphic and literary sources, and we can consider as proven the employment of this weapon in the praetorium throughout the 3rd century $\mathrm{AD}$ as a result of adaptation to new battle techniques in the face of old and new enemies.

\section{Palabras Clave - Keywords}

Arco; Castra Praetoria; cohortes pretorianas; ejército central de maniobra; pila; reforma severiana.

Arch; Castra Praetoria; Praetorian Cohorts; field army; pila; Severan reform.

El arco y las flechas no son armas conectadas usualmente con las cohortes pretorianas, pero ¿es cierta esta imagen?

La respuesta sería afirmativa para los siglos I y II d. C. ya que ninguna evidencia, ya sea artística, arqueológica o literaria nos indica el empleo del arco entre los pretorianos. Sin embargo, todo esto cambia al llegar el siglo III d. C. La primera prueba de ello la encontramos bajo el gobierno de Maximino el Tracio, aunque es probable que el cambio operase desde el gobierno de Caracalla.

A principios de abril del $238 \mathrm{~d}$. C. el pueblo romano había apoyado ya la fallida sublevación de Gordiano I y de Gordiano II en África y la plebe de Roma se oponía abiertamente al gobierno del emperador. Aprovechando esta coyuntura, un senador llamado Galicano y un expretor llamado Mecenas asesinan a varios soldados pretorianos que, desarmados, habían acudido a la entrada del senado con la intención de informarse sobre lo que éste decidía en momentos tan convulsos ${ }^{1}$.

* Doctor en Historia por la Universidad de Oviedo. Dirección: c/ Pintor Manuel Medina, 24, $5^{\circ}$ D, 33210, Gijón, Asturias. Email: cohorspraetoria@hotmail.com.

1 SHA, Los dos Maximinos, XX, 1-6; Her. VII, 11, 1-7. 
Los supervivientes son perseguidos y apedreados hasta los Castra Praetoria, en donde se refugian. Inmediatamente, Galicano inflama los ánimos de la plebe, convenciéndola para armarse, liberar a los gladiadores y así tomar el campamento y acabar con los pretorianos. Se inicia así un asalto descrito por Herodiano:

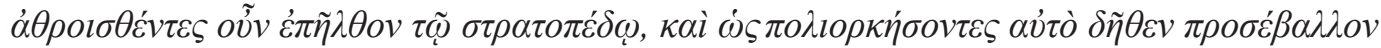

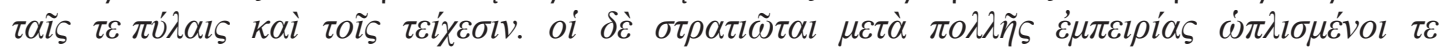

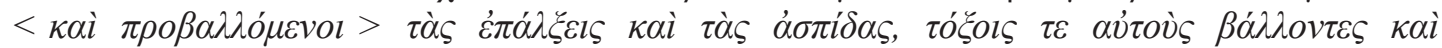

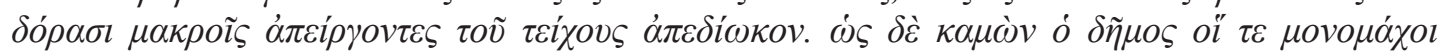

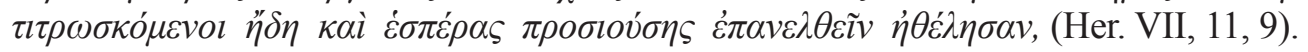

«El pueblo trastornado transformó en arma cualquier útil de un material adecuado que cayó en sus manos. Reunido, pues, aquel ejército, marcharon contra el campamento, y se lanzaron contra puertas y muros, con la evidente intención de tomarlo por asalto. Pero los soldados, con las armas de su dilatada experiencia y la protección de las almenas y escudos, rechazaron al pueblo de los muros disparando sus arcos y repeliéndolos con sus picas. Finalmente, al estar el pueblo cansado y los gladiadores heridos y acercándose ya la noche, decidieron retirarse».

Tras el fracaso del primer día el senado asumió la responsabilidad del combate efectuando levas en toda Italia y nombrando generales. Los siguientes días del asedio son narrados a continuación:

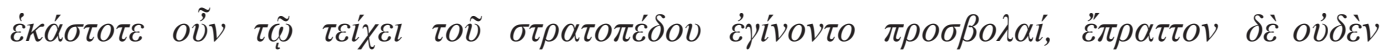

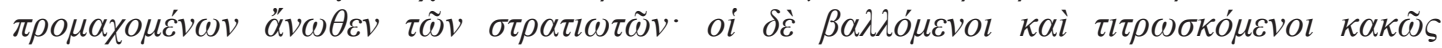
$\dot{\alpha} \pi \eta \lambda \lambda \dot{\alpha} \gamma \eta \sigma \alpha \nu$ (Her. VII, 12, 2).

«Todos los días se producían ataques contra los muros del campamento, pero sin ningún éxito. Los soldados desde su posición elevada rechazaban a los atacantes, que se retiraban maltrechos, heridos por las flechas y las picas».

La descripción de este suceso dada por Herodiano puede considerarse como la más fiable de todas las fuentes clásicas ${ }^{2}$. Sólo este autor menciona específicamente el uso de arcos y flechas por parte de los pretorianos, tanto en el primer ataque a los Castra Praetoria, por parte de la plebe, como en los sucesivos asaltos, llevados ya a cabo por las tropas del senado.

El empleo de esta arma está, evidentemente, provocado y condicionado por las circunstancias. En los Castra Praetoria sólo permanecían en estos momentos unos pocos hombres, missici probablemente ${ }^{3}$, que habían permanecido como reliquatio para el mantenimiento del campamento.

Su escaso número hizo necesario que tomasen todas las medidas a su alcance para defender el campamento y el uso de arco y flecha resultaba ideal a la hora de repelar y causar bajas a los atacantes desde la posición elevada que proporcionaba la muralla. Sin embargo, no existe constancia del uso de estas armas por las tropas del pretorio en el campo de batalla, así pues ¿por qué conocían su uso?, y ¿por qué las almacenaban en el arsenal del campamento?

La primera de las cuestiones resulta sencilla de responder: es la consecuencia de los nuevos tiempos. La llegada al poder de Septimio Severo marcó un cambio radical en la forma de entender el papel de las cohortes pretorianas en las campañas bélicas. Hasta entonces las

2 Ménard, 2004, p. 82.

3 Her. VII, 11, 2 especifica que eran soldados a punto de ser licenciados. 
campañas llevadas a cabo por el ejército romano no solían implicar el desplazamiento de más legiones completas que las situadas en el área inmediatamente próxima a la zona de conflicto. Los efectivos del ejército se completaban con vexillationes legionarias procedentes del resto de fronteras, que retornaban a su lugar de origen tras la campaña. De esta manera, aunque todo el sistema se debilitaba, se evitaba desguarnecer ningún punto de los limites.

Las carencias de este sistema quedaron en evidencia bajo el gobierno de Marco Aurelio. El sistema de vexillationes hacía que, ante un ataque simultáneo a varias fronteras (opción que se perfilaba como más probable cada vez) los refuerzos no pudieran ser desplazados de un lugar a otro, o que, sencillamente, tras haber sufrido un número importante de bajas las legiones no pudieran proporcionar vexillatio alguna.

Septimio Severo creyó necesaria la existencia de un ejército central de maniobra, mantenido normalmente en reserva, capaz de marchar a reforzar cualquier frontera, a repeler cualquier invasión o simplemente dispuesto para lanzar una ofensiva contra territorio hostil en cualquier momento que se creyese conveniente.

Este ejército central de maniobra, sobradamente conocido, estaba integrado por las cohortes pretorianas, la legión II Parthica, los equites singulares Augusti, y de ser necesario, las cohortes urbanas. Posteriormente se le añadieron otras tropas: los equites extraordinarii integrados por escitas y germanos bajo el gobierno de Caracalla ${ }^{4}$, y tropas de mauri y osroeni con Alejandro Severo ${ }^{5}$. Todas tropas de caballería para proporcionar el apoyo necesario a las cohortes pretorianas y los albani, unidades integradas en su mayor parte por infantería pesada.

La formación del ejército central de maniobra a partir de las cohortes pretorianas como núcleo principal trajo consigo ciertos condicionantes de cara al combate. Hasta este momento nunca habían marchado al combate más de entre dos y cinco cohortes pretorianas juntas ${ }^{6}$, y nunca actuando como unidad de primera línea, sino como reserva de élite para el caso de que las legiones fallasen.

A partir de este momento, con estos nuevos cambios, las cohortes pretorianas debían ser mucho más flexibles y adaptables, ya que la totalidad de soldados del pretorio podía ser llevada al combate en cualquier momento y contra cualquier enemigo. Obviamente, es sustancialmente distinto, por ejemplo, enfrentarse a los caledonios, con escasas protecciones corporales y grandes espadas de filo en un clima frío y lluvioso y una geografía montañosa, que a los persas sasánidas, con caballería pesada y arqueros a caballo en un clima desértico.

Este misma especialización queda patente también entre la legión II Parthica, en la cual además de constatar las especialidades de combate tradicionales de una legión, como la de jinete (CIL VI 3409 [p. 3393], AE 19931581 ó AE 1993 1583) o scorpio (AE 1993 1584)7, aparecen otras nuevas como la de lanciarius (AE 1993 1573, AE 19931574 y AE 1993 1575), la de phalangiarius ${ }^{8}$, y también la de arquero, esta última atestiguada en la misma época que los sucesos descritos por Herodiano:

- CIL VI 37262. Roma, Italia.

AVR(elio) MAIO C(ai) AR / TILI(o ?) EX SAGITTA / RIS (sic) SALARI(ari)VS / LEG(ionis) II PART(hicae) SEVE / RIANAE VIXIT AN / NIS LVI FILI [P]A / TRI (?) SVO B(ene) M(erenti) F(ecerunt)

4 Dión Cas. LXVIII, 5, 5.

De Laet, 1946-47, p. 540; Birley, 1969, p. 65.

Exceptuando la guerra civil del año 69 d. C. ya que Otón no disponía de más tropas.

Sinónimo de ballistarius.

Balty, 1988, pp. 100-101; Holder y Sumner, 1992, pp. 20-22; Ricci, 2000, pp. 400-401; Cowan y Mcbride, 2003, p. 24. 
«Aurelius Maius Artilius (?), (hijo) de Caius, de los arqueros, salararius de la legión II Parthica severiana, vivió 56 años. Lo hicieron los hijos al padre que bien lo merecía».

Es decir, la legión II Parthica era en sí misma un pequeño ejército con tropas de todo tipo: infantería pesada, infantería ligera, caballería, artillería y arqueros. De esta manera podían no sólo afrontar cualquier enemigo, sino actuar en vexillationes independientes perfectamente adecuadas para cada misión específica. Recordemos que Vegecio recomienda la instrucción de los legionarios, al menos de una parte de ellos, en el manejo del arco por lo que pudiera suceder en combate?

Hay que suponer que las cohortes pretorianas, probablemente sin llegar al extremo de la legión II Parthica, sufrieron un proceso similar, que ya no habría de detenerse hasta la desaparición de éstas. Encontramos prueba de ello en el siguiente epígrafe:

- CIL VI 3595 (pp. 837 y 3407) = AE 1971209 = AE 1998154 = PEREA YÉBENES, S.; «Un cipo sepulcral de Roma en Madrid y los doctores - evocati. Nueva interpretación de CIL VI 3595», Gerión 16 (1998), pp. 271-306.

\section{$\mathrm{D}$ (is) M(anibus) / T(ito) FLAVIO / EXPEDITO / DOCTORI / SAGITTAR(um) / FLAVIA / EVPHROSYME / ET / ATTICA FILIAE / PATRI B(ene) M(erenti)}

«A los dioses Manes. A Titus Flavius Expeditus, doctor de los arqueros. (Lo hicieron) Flavia Euphrosyme y Attica, hijas, al padre que bien lo merecía».

Este epígrafe puede fecharse entre el último cuarto del siglo III d. C. y las primeras décadas del siglo IV d. C. ${ }^{10}$ Titus Flavius Expeditus es, a todas luces, un euocatus de las cohortes pretorianas ${ }^{11}$. Perea Yébenes opina que, dado que las cohortes pretorianas no poseían arqueros, lo más probable era que Flavius Expeditus no fuese instructor de alguna tropa de arqueros, sino que se trataba de un experto en la fabricación de flechas con destino al armamentarium de las scholae palatinae ${ }^{12}$. Sin embargo, como ya hemos visto esto no tiene porqué ser cierto.

La primera objeción que podría hacerse a esta teoría es que no parece adecuado dejar en manos de un ex-pretoriano la fabricación de las armas destinadas a la tropa que reemplazó a las cohortes pretorianas. Máxime, si como se venía creyendo, los pretorianos no tenían conocimiento alguno en el uso del arco. Esta teoría resulta forzada, y lo es, ya que se produce una pequeña confusión.

La impresión primera de Perea Yébenes, que Flavius Expeditus era un antiguo pretoriano, se ve influenciada por la obra de Rankov ${ }^{13}$, corrigiendo en consecuencia la que fue su idea original. Sin embargo, Rankov realiza una obra breve y esencialmente concentrada en los siglos I y II d. C. ${ }^{14} \mathrm{e}$ ignora casi por completo las peculiaridades de las cohortes pretorianas tras la reforma severiana.

Aunque en efecto las cohortes pretorianas no poseían arqueros, los soldados pretorianos recibían instrucción en el manejo del arco a fin de cubrir una posible eventualidad. Ésta era la tarea de Flavius Expeditus en los últimos momentos de las cohortes pretorianas.

Una situación similar se producía con respecto al uso de la artillería. Sabemos que durante el gobierno de Majencio la muralla de los Castra Praetoria fue dotada de nuevas torres de ladrillo con acceso a la parte superior mediante grandes puertas de arco preparadas para per-

\footnotetext{
9 Veg. I, 15 y II, 23.

10 Perea Yébenes, 1998, pp. 300-304.

11 Perea Yébenes, 1998, pp. 292-296.

12 Perea Yébenes, 1998, pp. 297-298.

13 Perea Yébenes, 1998, pp. 298, nota 99.

14 Rankov y Hook, 1994, pp. 18-27.
} 
mitir el paso de máquinas de guerra ${ }^{15}$ con que repeler un posible asalto. Por lo tanto, en los primeros años del siglo IV d. C., los soldados pretorianos estaban capacitados para el uso de piezas artilleras, a pesar de que las cohortes pretorianas nunca en su historia dispusieron de artillería ni la utilizaron ${ }^{16}$.

La existencia de arcos compuestos en el arsenal de los Castra Praetoria ha de vincularse también con la presencia de jinetes osroenos y escitas, integrados en la guarnición urbana desde el gobierno de Caracalla ${ }^{17}$. Estas tropas, compuestas por caballería ligera, apoyaban en campaña a los pretorianos y probablemente hacían un uso intensivo del arco.

El empleo del arco no es la única especialización desarrollada por las cohortes pretorianas. Constatamos la aparición de soldados especializados en el uso de la lancea (CIL VI 2759 [p. 3370 y 3835] = ILS 2045; ILS 2782; CIL VI 2787a) al mismo tiempo que los relieves tallados en los epígrafes pretorianos muestran los soldados equipados con el pilum a lo largo de todo el siglo III d. C. Los pila mostrados, además de estar profusamente adornados, aparecen sin lastre alguno (CIL VI 2730 [p. 3370] = AE 197920 = AE 1996 91), lastrados con un único lastre (AE 1990752 = AE $1992579=$ AE 1994 606) o con dos (CIL VI 2602 [p. 3369]). Este uso de pila doblemente lastrados es de hecho exclusivo del pretorio. En un momento en que la lancea sustituye progresivamente al pilum en las legiones ${ }^{18}$ los pretorianos no sólo emplean ambas armas, sino que evolucionan el pilum adecuándolo a un uso muy especializado. A mayor peso del pilum se disminuía el alcance y se incrementaba la capacidad de penetración, lo que lleva a pensar que las cohortes pretorianas se especializaron en luchar contra enemigos pesadamente acorazados.

Podemos concluir que la combinación de arco, lanceae y de los distintos tipos de pila permitía a los soldados de las cohortes pretorianas batir eficazmente a cualquier tipo de enemigo, fuese cual fuese su armadura, a distancias de entre 150 metros (con los arcos) ${ }^{19}$ y 30 metros $(\text { con los pila })^{20}$, e incluso formar una falange si era necesario. Del mismo modo permitía a los soldados pretorianos defender un campamento o una ciudad amurallada, u hostigar a un enemigo que se halle en una posición elevada.

Este hecho otorgó a las cohortes pretorianas, al igual que a la legión II Parthica, una capacidad de respuesta y una disponibilidad a todos los niveles que no había existido hasta entonces en el ejército romano. El ejército central de maniobra se convirtió en una herramienta imprescindible en las ofensivas romanas en cualquier parte del imperio estando siempre capacitado para la operación.

\section{BIBLIOGRAFÍA}

Fuentes clásicas:

Herodiano, Historia del imperio romano después de Marco Aurelio. Traducción de J. J. Torres Ebarranch, Madrid, 1985 (ed. Gredos).

Herodian in two volumes. Traducción al inglés de C. R. Whittaker, Londres, 1969 (ed. Loeb).

\footnotetext{
15 Menéndez Argüín, 2006, pp. 62-63.

16 Ceñal Martínez, 2009, pp. 250-251.

17 De Laet, 1946 - 47, pp. 539- 540.

18 Menéndez Argüín, 2000, p. 143.

19 Quesada Sanz, 2008, p. 122.

20 Cowan y Mcbride, 2003, p. 26.
} 


\section{Bibliografia moderna:}

Balty, J. CH. (1988): «Apamea un Syria in the second and third centuries A. D.», Journal of Roman Studies LXXVIII, pp. 91-104.

Birley, E. (1969): «Septimus Severus and the Roman Army», Epigraphische Studien VIII, pp. 63-82.

Ceñal Martínez, H. (2009): La guardia pretoriana. Composición, funciones e historia. Tesis doctoral no publicada dirigida por el Prof. Dr. Narciso Santos Yanguas, catedrático de Historia Antigua de la Universidad de Oviedo, leída el 15 de enero de 2009.

Cowan, R. y McBride, A. (2003): Imperial Roman legionary AD 161-284, Oxford, Osprey Publishing.

Holder, P. A. y Sumner, G. (1992): «Legio II Parthica: A third century Legion», Military Illustrated 48, pp. 18-23.

De Laet, S. J. (1946-47): «Les pouvoirs militaires des préfets du prétoire et leur développement progressif», Revue Belge de Philologie et d'historie 25, pp. 509-554.

Ménard, H. (2004): Maintenir l'ordre à Rome (IIe - IVe AP. J. - C.), Seyssel, Ed. Champ - Vallon.

Menéndez Argüin, A. R. (2000): Las legiones del s. III d. C. en el campo de batalla, Écija, Editorial Gráficas Sol.

Menéndez Argüin, A. R. (2006): Pretorianos. La guardia imperial de la antigua Roma, Madrid, Almena Ediciones.

Perea Yébenes, S. (1998): «Un cipo sepulcral de Roma en Madrid y los doctores - evocati. Nueva interpretación de CIL VI 3595», Gerión 16, pp. 271-306.

Quesada Sanz, F. (2008): Armas de Grecia y Roma. Forjaron la historia de la Antigüedad clásica, Madrid, La Esfera de los Libros.

Rankov, B. (1994): The Praetorian Guard, Oxford, Osprey Publishing.

Ricci, C. (2000): «Legio II Parthica. Una messa a punto» en Les légions de Rome sous le Haut - Empire. Actes du Congrès de Lyon (17-19 septembre 1998) rassemblés et édités par Y. Le Bohec avec la collaboration de C. Wolf, Lyon, pp. 397-406.

Recibido: $19 / 05 / 2010$

Aceptado: 03/11/2011 